\title{
HSA-based anti-inflammatory therapy: a new and improved approach
}

“HSA holds great potential as a drug carrier for various life-threatening diseases, including inflammation and cancer."

Keywords: anti-inflammatory therapy $\approx$ delivery system $\approx$ human serum albumin $\approx$ interactive associations of drugs

Anti-inflammatory drugs, which typically contain steroids and nonsteroidal anti-inflammatory drugs, are widely used to ameliorate pain by combating inflammation. In fact, the earliest anti-inflammatory drugs are from the serendipitous discovery of certain plants and their extracts being applied for the relief of pain, fever and inflammation. However, since aspirin was discovered in 1897 , many anti-inflammatory drugs have been developed and marketed. These agents are frequently prescribed for the management of arthritis symptoms, including joint pain and inflammation. Unfortunately, although anti-inflammatory drugs have good therapeutic efficacy, they are known to be associated with a broad spectrum of side effects, including gastrointestinal and cardiovascular events, renal toxicity, increased blood pressure, and deterioration of congestive heart failure [1]. Thus, we have been trying to find new anti-inflammatory drugs with improved efficacy and safety. So far, clinically meaningful differences in the therapeutic efficacy of different anti-inflammatory drugs have not been reported. Therefore, it is imperative to develop new strategies in order to design antiinflammatory drugs that are less toxic but still treat inflammation as effectively.

Human serum albumin (HSA), is a ubiquitous serum protein with an expansive list of associated endogenous and exogenous compounds. There has been research to identify many drugs that display altered pharmacokinetic and pharmacodynamic properties due to interactions with HSA [2]. In addition, HSA, due to its unique biochemical and pharmacological characteristics, has been used as a versatile drug carrier, resulting in higher efficacy of treatment and reduced side effects $[3,4]$. In our opinion, HSA-based delivery systems and HSA structure-guided design prodrugs may be a promising strategy for treatment of inflammation.

\section{HSA-based design of anti-inflammatory prodrugs}

Aspirin is the most widely used anti-inflammatory drug in the world. It is a nonselective and irreversible cyclooxygenase inhibitor, and prevents the production of thromboxane A2 in platelets by acetylating the serine-529 residue of the cyclooxygenase-1 isoform. Furthermore, the cardioprotective properties of aspirin are well established; indeed, it forms the cornerstone of primary and secondary prevention in ischaemic vascular disease through inhibition of platelet aggregation. Unfortunately, aspirin confers many side effects that have been well-characterised. These side effects include peptic ulceration, upper gastrointestinal bleeding, and haemorrhagic stroke with routine aspirin administration. These lead it to have a significant amount of morbidity and mortality, making the need for a new type of drug evident.

Diflunisal (FL) is an exciting new asprinlike nonsteroidal anti-inflammatory drugs that blocks the activity of the prostaglandin synthesizing enzyme cyclooxygenase-2. It has a higher potency, in vivo tolerance and longer duration of action compared with aspirin [5]. However, FL is not perfect. It is $99 \%$ protein-bound in plasma, requiring two $250 \mathrm{mg}$ doses every day for sufficient efficacy [6]. This is not only expensive, but while at lower doses, FL does not generally cause serious side effects, it does cause gastrointestinal irritation when given chronically at a high dose. Therfore, modifying difunisal to have reduced affinity for serum albumin would be highly beneficial for lower dosage and higher in vivo tolerance.

$\mathrm{FL}$, with strong binding affinity, interacts with the IIA and IIIA subdomains of HSA [7,8]. Promising previous research has been done synthesizing analogs of FL that reduce binding to the IIIA domain, which has resulted in a tenfold decrease in affinity between the analogs and

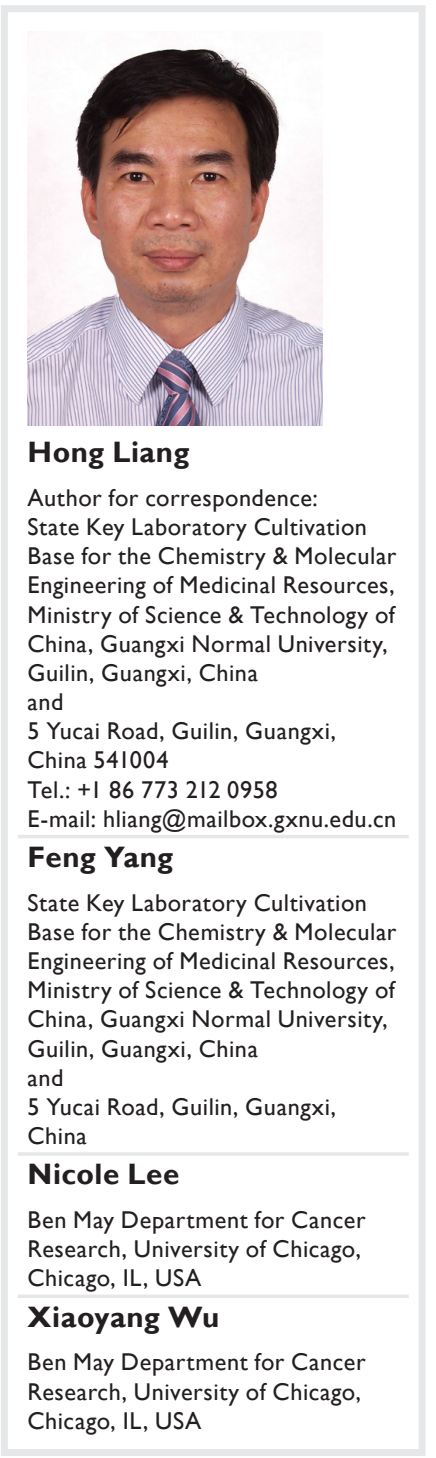


HSA [8]. However, in our opinion, subdomain IIA of HSA is the most promising binding site for future FL development because of the following two main reasons.

There are three main strong binding sites on HSA for fatty acid (FA) interaction. Of those three, one is located on the IIIA subdomain [9]. FAs compete with drugs when binding to HSA in vivo. This is clear because nonesterified FAs are the primary endogenous molecules that usually bind to HSA, and in normal physiological conditions in the plasma, every HSA molecule usually binds up to two FA molecules. This number can go up to six in certain disease states. Analysis of the structures of HSA-FA and drug complexes has shown that FAs prevent drugs from binding to subdomain IIIA, but drugs predominantly displace FA binding to subdomain IIA since this interaction is weaker.

HSA can not only bind a wide variety of drugs, but also possesses important enzymatic activity mainly due to the presence of two reactive residues; Lys199 in subdomain IIA and Tyr411 in subdomain IIIA. HSA is one of the major enzymes in plasma capable of hydrolyzing ester prodrugs to their pharmacologically active forms $[11,12]$. Previous results revealed that Lys199 takes part in a nucleophilic substitution reaction with some drugs bound to the IIA subdomain, which causes the binding mode of the drugs to change $[13,14]$. Thus, if $\mathrm{FL}$ is redesigned into the prodrugacetyldiflunisal (AFL), the high dosage required may be able to be reduced. As a matter of fact, the results agree with our hypothesis. This is because FL is released from AFL after the Lys199 residue on HSA undergoes nucleophilic attack on the acetyl group [7]. Comparing with the binding affinity of FL directly to the IIA subdomain of HSA, the binding affinity between FL released from AFL and HSA is lessened, since their binding modes are different. In HSA-FA-AFL, the hydroxyl group on FL forms a hydrogen bond with Arg222 (with a bond length of $3.30 \AA$ ), and the carboxylate group on FL forms another hydrogen bond with acetyllysine-199 (3.41 Å) [7]. For comparison, in HSA-FA-FL, there is the formation of three bonds - two hydrogen bonds with Lys199 (3.30 $\AA$ ) and His242 (3.01 $\AA$ ), and one salt bridge with Lys199 (2.85 $\AA$ ) [7]. This decrease in binding affinity is twofold. This means that by using AFL, a much lower dosage of drugs will be necessary to treat inflammatory pain. These results not only provide a method for the rational design of HSA-based prodrugs, but also pave the way for the effective application of FL in the clinic.

\section{HSA-based approach to} anti-inflammatory therapy

HSA-based delivery systems are promising for improving drug targeting and reducing negative side effects $[2,3]$. In the clinical scene, albumin is becoming more and more prominent in its use as a drug carrier. There are three primarily accepted drug-delivery techniques: conjugation with bioactive proteins, coupling of both endogenous and exogenous albumin with drugs that have a lowmolecular-weight, and encapsulation and packaging of drugs in albumin nanoparticles [15]. We can make HSA as a carrier to bind or conjugate anti-inflammatory drugs for inflammation treatment. To synergistically treat inflammation, we may use HSA to deliver several different drugs via the three main different binding sites (the IB, IIA and IIIA subdomains) at the same time.

It is possible to make several drugs bind with the same binding site at the same time since there is a large hydrophobic cavity at the IIA subdomain $[10,16]$. Therefore, modification of the IIA subdomain may be a promising strategy to enhance HSA binding and delivery of drugs when we want to design HSA to be a carrier for drugs that can not bind with HSA. For example, the most cationic drugs poorly associate with HSA at the IIA subdomain, but we can modify the binding environment of the IIA subdomain by other anionic drugs, which regulate cationic drugs to bind here [17]. It raises the tempting possibility to fully exploit the unique binding capacity of HSA's IIA subdomain to achieve simultaneous delivery of anionic and cationic drugs.

In addition, several anti-inflammatory drugs are often simultaneously used to treat inflammation in a clinical setting. To achieve the optimal effect, there exists a challenge where co-binding drugs at the same binding site can influence each other's binding affinities. In fact, when several drugs co-bind to the IIA subdomain of HSA, a potential change to the drugs' binding positions and modes to each other may occur. Meanwhile, those changes regulate the respective drugs' binding affinities accordingly [18]. It raises the intriguing possibility of using HSA as a multidrug carrier by changing the binding environment of the IIA subdomain, and even controlling a drug's release speed by drug self-regulation.

Currently, carbon nanotubes, acting as either the main drug carrier or as adjuncts to assist or modify their release from another parent delivery system, have been extensively studied to deliver anti-inflammatory drugs [19]. Utilizing 
HSA as a delivery system for drugs has many beneficial characteristics. HSA is a nontoxic and nonantigenic endogenous protein that can carry different hydrophobic and hydrophilic drugs throughout blood circulation. What is more, drugs binding with HSA directly can form a 'nano-drug', increasing drug bioavailability [20]. Therefore, we can design and develop multifunctional HSA nanotubes as anti-inflammatory drug carriers, modulate drug release and avoid toxicity produced by other nanotubes.

In summary, HSA holds great potential as a drug carrier for various life-threatening diseases, including inflammation and cancer. Delineation of HSA structure in complex with different compounds will be essential for us to understand its biochemical behavior in vivo and design more effective therapeutic strategy in the future.

\section{Financial \& competing interests disclosure}

The authors would like to thank the financial support from the Natural Science Foundation of China (31060121, 21171043) and the Natural Science Foundation of Guangxi (2012GXNSFCB053001, 2013GXNSFGA019010). The authors have no other relevant affiliations or financial involvement with any organization or entity with a financial interest in or financial conflict with the subject matter or materials discussed in the manuscript apart from those disclosed. No writing assistance was utilized in the production of this manuscript.

\section{References}

1 Ong CK, Lirk P, Tan CH, Seymour RA. An evidence-based update on nonsteroidal antiinflammatory drugs. Clin. Med. Res. 5, 19-34 (2007).

2 Peters T. All about Albumin: Biochemistry, Genetics, and Medical Applications. Academic Press, San Diego, CA, USA (1995).

3 Neumann E, Frei E, Funk D et al. Native albumin for targeted drug delivery. Expert Opin. Drug Deliv. 7, 915-925 (2010).

4 Elsadek B, Kratz F. Impact of albumin on drug delivery - new applications on the horizon. J. Control. Release 157, 4-28 (2012).

5 Hannah J, Ruyle WV, Jones $\mathrm{H}$ et al. Discovery of diflunisal. Br. J. Clin. Pharmacol. S1, 7-13 (1977).

6 Gilman AG, Rall TW, Nies AS, Taylor P. The Pharmacological Basis of Therapeutics. Pergamon Press, NY, USA (1990).

7 Yang F, Ma ZY, Zhang Y et al. Human serum albumin-based design of a diflunisal prodrug. Eur. J. Pharm. Biopharm. 84, 549-554 (2013).

8 Mao H, Hajduk PJ, Craig R, Bell R, Borre T, Fesik SW. Rational design of diflunisal analogues with reduced affinity for human serum albumin. J. Am. Chem. Soc. 123, 10429-10435 (2001).
9 Simard JR, Zunszain PA, Ha CE et al. Locating high-affinity fatty acid-binding sites on albumin by x-ray crystallography and NMR spectroscopy. Proc. Natl Acad. Sci. USA 102, 17958-17963 (2005).

10 Ghuman J, Zunszain PA, Petitpas I, Bhattacharya AA, Otagiri M, Curry S. Structural basis of the drug-binding specificity of human serum albumin. J. Mol. Biol. 353, 38-52 (2005).

11 Yang F, Bian C, Zhu L, Zhao G, Huang Z, Huang M. Effect of human serum albumin on drug metabolism: structural evidence of esterase activity of human serum albumin. J. Struct. Biol. 157, 348-355 (2007).

12 Liyasova MS, Schopfer LM, Lockridge O. Reaction of human albumin with aspirin in vitro: mass spectrometric identification of acetylated lysines 199, 402, 519, and 545. Biochem. Pharmacol. 79, 784-791 (2010).

13 Kragh-Hansen U. Molecular and practical aspects of the enzymatic properties of human serum albumin and of albumin-ligand complexes. Biochim. Biophys. Acta 1830(12), 5535-5544 (2013).

14 Wang Y, Yu H, Shi X, Luo Z, Lin D, Huang M. Structural mechanism of ring-opening reaction of glucose by human serum albumin. J. Biol. Chem. 288, 15980-15987 (2013).

15 Kratz F. Albumin as a drug carrier: design of prodrugs, drug conjugates and nanoparticles. J. Control. Release 132, 171-183 (2008).

16 Zhu L, Yang F, Chen L, Meehan EJ, Huang M. A new drug binding subsite on human serum albumin and drug-drug interaction studied by x-ray crystallography. J. Struct. Biol. 162, 40-49 (2008).

17 Yang F, Lee P, Ma Z et al. Regulation of amantadine hydrochloride binding with IIA subdomain of human serum albumin by fatty acid chains. J. Pharm. Sci. 102, 84-92 (2013).

18 Yang F, Yue J, Ma L et al. Interactive associations of drug-drug and drug-drugdrug with IIA subdomain of human serum albumin. Mol. Pharm. 9, 3259-3265 (2012).

19 Fanali G, di Masi A, Trezza V, Marino M, Fasano M, Ascenzi P. Human serum albumin: from bench to bedside. Mol. Aspects Med. 33, 209-290 (2012).

20 Wong BS, Yoong SL, Jagusiak A et al. Carbon nanotubes for delivery of small molecule drugs. Adv. Drug Deliv. Rev. doi:10.1016/j. addr.2013.08.005 (2013) (Epub ahead of print). 